\title{
SCFT Simulations of Thin Film Blends of Block Copolymer and Homopolymer Laterally Confined in a Square Well
}

\author{
Su-Mi Hur \\ Department of Chemical Engineering
}

Carlos J. García-Cervera

Department of Mathematics

\author{
Edward J. Kramer and Glenn H. Fredrickson \\ Departments of Chemical Engineering and Materials
}

\author{
University of California, Santa Barbara, \\ Santa Barbara, CA, 93106
}

May 1, 2009

\begin{abstract}
Recently there has been significant interest in manipulating the self-assembly behavior of block copolymers to obtain structures that are not observed in the bulk. Here we explore the conditions for which self-assembly in laterally confined thin block copolymer films results in tetragonal square arrays of standing up cylinders. More specifically, we used self-consistent field theory (SCFT) to study the equilibrium phase behavior of thin films composed of a blend of $\mathrm{AB}$ block copolymer and A homopolymer laterally confined in square wells. By using suitable homopolymer additives and appropriately sized wells, we observed square lattices of upright B cylinders that are not stable in pure
\end{abstract}




\begin{abstract}
$\mathrm{AB}$ block copolymer systems. We further investigated the optimal conditions and parameters that lead to defect-free, in-plane tetragonal ordering. Considering the potential application of such films in block copolymer lithography, we also conducted numerical SCFT simulations of the role of line edge roughness at the periphery of the square well on feature defect populations. Our results indicate that the tetragonal ordering observed under square confinement is robust to a wide range of boundary perturbations.
\end{abstract}

\title{
1 Introduction
}

Over the past half century there has been significant progress in reducing both the size and associated fabrication cost of microelectronic circuit devices. However, since the 1990s, technical and economic limitations associated with optical lithography have been predicted due to the cost and difficulties in decreasing the irradiation wavelength. ${ }^{1}$ Thus in the past decade, the self-assembly of block copolymer thin films has attracted considerable attention as a promising high resolution lithographic tool. The nanometer scale of microdomain ordering and its facility for modulation of size and pattern ${ }^{2-7}$ renders block copolymer thin films a particularly promising candidate as lithographic masks for the creation of next generation information storage and electronic devices. Among the structures that can be produced with such masks are regular arrays of quantum or magnetic dots, membranes with tailored nanoscale porosity, and dense assemblies of nanowires. ${ }^{1,8}$

One challenge for block copolymer lithography, and for all advanced lithography strategies that invoke self-assembly, is the creation of robust, defect-free feature arrays. Moreover, in order to fabricate non-periodic features such as bent lines with sharp corners, lines that end at specific positions, T-junctions, jogs, and non-periodic arrays of dots, it is necessary to devise confinement or patterning strategies for block copolymer films that disrupt, modify, or truncate the periodic copolymer microdomains that occur naturally in the bulk. In some cases, it is also desirable to produce periodic arrays of features, but with a lattice symmetry that differs from the usual hexagonal close-packed arrays commonly observed in both thin films and bulk samples of AB block copolymers. Square arrays of microdomains are particularly attractive, since they represent a canonical microlithographic structure that is consistent with the industry standard integrated circuit design architecture 
for addressability and transistor interconnection.

Controlling and perfecting the microdomain order and orientation is of paramount importance in block copolymer lithography since defects can decrease device yield. There are various approaches to controlling long-range (or global) in-plane order. ${ }^{5}$ One method involves applying one or more external fields such as an electrical, flow, or thermal gradient field, to a sample. Another approach involves the use of templated substrates, where a chemically patterned surface serves to direct the self-assembly process. ${ }^{9-13}$ A third method for controlling and improving long-range microdomain order is graphoepitaxy, which uses a topographically patterned substrate. ${ }^{7,14-19}$ Graphoepitaxy integrates bottom-up self-assembly of block copolymers on 10 nm scales with top-down conventional lithography in generating micron-scale wells by which lateral confinement can promote defect-free self-assembled block copolymer features at low cost. Segalman et al. ${ }^{14}$ demonstrated that a planar wall significantly increased the lateral ordering of spherical microdomains in block copolymer films deposited on substrates topographically patterned with grooves. Sundrani et al. ${ }^{15}$ and Hammond et al. ${ }^{17}$ showed that cylinders of diblock copolymer are guided to align by a preferential wetting layer on the vertical sidewalls of a lithographical channel. Stein et $\mathrm{al}^{19}$ and Bita el $\mathrm{al}^{20}$ observed long-ranged hexagonal lattices of spheres in block copolymer films on more general topographically patterned templates including hexagonal wells, while Xiao et $\mathrm{al}^{18}$ observed long-ranged hexagonal order of standing up cylinders confined to a neutral channel. On the theoretical side, a two-dimensional self-consistent field theory (SCFT) simulation study was presented by Bosse et al. ${ }^{21}$ for an $\mathrm{AB}$ diblock copolymer thin film laterally confined in a hexagonal-shaped well.

While there has been significant research aimed at improving the longrange order of hexagonal arrays, less attention has been paid to the fabrication of square lattices by means of block copolymer assembly. Park et al. demonstrated that a well-ordered square array of cylinders can be formed on a chemically patterned surface from a block copolymer system whose bulk behavior consists of a hexagonal array of cylinders. ${ }^{22}$ However, the viability of such a scheme is potentially limited by the high cost of the nanoscale lithography required to generate the chemically patterned substrate. A second strategy for achieving square (i.e. "tetragonal") lattices of cylinders or spheres invokes the natural tendency of $\mathrm{ABC}$ triblock copolymers to form such structures. In particular, compositionally symmetric $\mathrm{ABC}$ triblocks with strongly repulsive $\mathrm{A}$ and $\mathrm{C}$ blocks are known to exhibit tetragonal mi- 
crophases. ${ }^{23}$ A disadvantage of this strategy is that it requires the rather challenging synthesis of ABC triblocks. Tang et al. ${ }^{24}$ recently demonstrated an alternative route to tetragonal order that involves the use of a binary blend of $\mathrm{AB}$ and $\mathrm{B}$ 'C diblock copolymers in which the $\mathrm{B}$ and $\mathrm{B}$ ' blocks have attractive supramolecular interactions. The self-assembly characteristics of this blend are similar to those of a corresponding ABC triblock system, but its realization requires a less demanding synthesis of two diblock copolymers. Nonetheless, graphoepitaxial strategies to control and improve long-range tetragonal order in such a system with three or more chemically distinct blocks have yet to be developed.

In the present paper, we investigate an alternative approach to achieving defect-free tetragonal arrays of microdomains that involves subjecting a simpler AB block copolymer system to graphoepitaxial confinement in a

square well. Specifically, by means of self-consistent field theory (SCFT) simulations, we show that it is possible to achieve square ordering in a blend of an $\mathrm{AB}$ diblock copolymer and an A homopolymer confined in a square well, provided that molecular weights and concentrations of the components are carefully selected. The mechanism for stabilization of tetragonal order is the alleviation of stress in the A blocks of the copolymer by the A homopolymer occupying regions in the microstructure that otherwise would be filled by highly stretched copolymer segments. ${ }^{25}$ Here we report on an SCFT study of phase morphology in square-confined $\mathrm{AB} / \mathrm{A}$ blends upon varying a number of parameters including the composition of the blend, the size of the square confinement well, and the wetting conditions that are imposed on the perimeter of the well. We identify optimal sets of parameters that promote the formation of a defect-free tetragonal array that spans the square well. Furthermore, by comparing the free energy of various observed and possible configurations, including kinetically trapped metastable configurations, we have constructed phase diagrams that will assist the design of experimental systems. Finally, we report on SCFT simulations of microdomain ordering within square wells subject to boundary perturbations in order to confirm the robustness of tetragonal ordering to line edge roughness.

\section{Model and Numerical Methods}

In this study, the model equations for a blend of AB block copolymers and $\mathrm{A}$ homopolymers in confinement is built on the standard Gaussian-chain model, 
with a Flory-type monomer-monomer interaction $\chi$ describing the strength of interactions between dissimilar segments and an explicit incompressibility constraint. A particle-based model for a system of interacting polymer chains is converted into a field-based model of a single chain in an external field through formally exact methods related to the Hubbard-Stratonovich transformation. The resulting statistical field theory is simplified by imposing a mean-field approximation, resulting in the well known equations of self-consistent field theory (SCFT) ${ }^{26,27}$ Here we consider a square domain of side length $L$ (in units of the unperturbed radius of gyration of an AB diblock copolymer, $R_{g}$, typically on the order of $3 \sim 20 \mathrm{~nm}$ ), which is discretized using spectral collocation on a square lattice with $N_{x}=N_{y}$ points in the $x$ and $y$ directions, respectively. The lattice spacing was chosen to be $0.25 R_{g}$, which provides sufficient numerical accuracy for the present study.

The four boundaries of the square cell are assumed to have an affinity for $\mathrm{A}$ and $\mathrm{B}$ segments that is tunable, but homogeneous along the perimeter. We also assume that the system is homogeneous in the $z$-direction. An experimental realization of such a system could be obtained by the "liftoff" technique ${ }^{28}$ or by use of a bilayer substrate. In the lift-off method, a resist is patterned in a square shape onto a neutral surface using optical or e-beam lithography, then material that has selectivity for A or B segments is deposited onto the patterned resist. After removing the resist, the material not on the resist remains to form mesas surrounding the well and its sides attract either the A or B blocks. In the second approach of using a bilayer substrate, by etching through just the top layer to define a square well, the four sides of the well would have selectivity for A and B segments set by the surface chemistry of the top layer, while the chemistry of the bottom layer would dictate the selectivity of the bottom surface of the square well. For both methods, by choosing the bottom layer appropriately, e.g. a random copolymer of A and B segments, it should be possible to create a neutral bottom surface. Assuming that the top surface could also be neutralized, such a strategy would in principle allow square wells to be created, which when filled with block copolymer and annealed, should produce 2D composition variations restricted to the $x-y$ plane.

The derivation of the model equations used in this study was detailed in a previous paper by Bosse et al. ${ }^{21}$ the key equations and numerical methods are summarized in the appendix. 


\subsubsection{Sine basis simulations}

Previous SCFT simulations of confined block copolymers ${ }^{21,29}$ used a masking method to model confinement, in which the modified diffusion equations (eq 14) are solved using a spectral or pseudo-spectral method with a Fourier series expansion and periodic boundary conditions. The mask is imposed within the simulation domain by specifying a polymer density function that vanishes outside the physical domain and rises smoothly away from the domain boundaries to the bulk polymer density $\rho_{0}$ inside the physical domain. An attractive feature of the masking method is that it is highly adaptable to different confinement geometries, whether regular or irregular in shape. However, it leads to a reduction in computational efficiency because it is necessary to resolve and compute thoughout the entire simulation domain, which extends outside of the physical domain. For physical domains of simple shapes, such as the square domain considered here, we can reduce the computational effort by simply compressing the mask to the simulation domain boundary (so that the physical and simulation domains are coincident) and imposing homogeneous Dirichlet, rather than periodic, boundary conditions on the modified diffusion equations of the theory. We further replace the specified polymer mask density $\rho_{p}(\mathbf{x})$ with a corresponding "wall" density $\rho_{w}(\mathbf{x}) \equiv \rho_{0}-\rho_{p}(\mathbf{x})$, which is set to $\rho_{0}$ at the boundary and smoothly decays to zero inside the domain.

Dirichlet boundary conditions can be conveniently imposed in a Fourier spectral collocation framework through the use of sine basis functions. There are several advantages to using a sine basis over plane-waves: decreased computational requirements by applying a real sine transform rather than the complex Fourier transform, no wasted computations in the masked-out wall areas, and less required resolution and faster convergence with respect to self-consistent field updates. The semi-implicit-Seidel(SIS) field update scheme summarized in eq 20 typically offers faster convergence and improved stability than explicit relaxation methods in simulations of polymer melts without confinement. ${ }^{30}$ However, for a confined system with a density mask and a plane wave basis, the pressure-like field $\left(W_{+}\right)$has a large-amplitude, rapidly varying feature at the mask boundary that dramatically slows the convergence of SIS updates. By moving the mask boundary to the edge of the computational domain and employing a sine wave basis, we have found that the pressure anomaly at the wall can be suppressed, thus allowing for larger time steps and more rapid convergence of SIS updates. 
In order to validate the performance of our sine basis algorithm, we have numerically investigated the convergence rates of both the explicit forward Euler (eq 19) and the SIS field update (eq 20) schemes applied to the SCFT equations for both the plane wave masking and the sine basis methods. Our test model was a one-dimensional slab of a symmetric AB diblock copolymer melt (fraction of A segments in the $\mathrm{AB}$ diblock, $f=0.5$ ) with $\chi N=16$, where $\chi$ is the A-B Flory interaction parameter and $N$ is the number of statistical segments on the copolymer. The confining walls, defined by a mask function $\rho_{w}(x)$ with a smooth hyperbolic tanh form (exact function is specified in the appendix), was made attractive to B segments imposing an appropriate wall $\chi$ parameter, $\chi_{w} N=-\left(\chi_{w A} N-\chi_{w B} N\right) / 2=-17$. Fig. 1 shows the converged $W_{ \pm}$fields of this confined system for (a) masking using a plane wave basis, and (b) a sine basis simulation. Fig. 2 shows the improved convergence of the sine-basis method by plotting the error, defined as the $l_{1}$ norm of the field-gradients of the Hamiltonian, i.e. eqs 17 and 18, at each iteration step. The time step for each of the four cases was taken as the maximum allowable for stable reduction of the error.

As shown in Fig. 1(a), when we use a masked simulation domain with a plane wave basis, the pressure-like $W_{+}$field shows sharp peaks near the walls that hinder the use of large time steps in the SIS field update. Because of this pressure anomaly, there is no advantage to using SIS over explicit methods for field updates since both methods exhibit similar convergence characteristics and the SIS scheme is approximately twice as expensive per iteration. Thus SIS for a confined system using the masking method is not as efficient as it is for bulk simulations, both simulations conducted with plane waves. However, by using a sine basis, where the masked area is confined to the interfacial region immediately adjacent to the walls, a different behavior is observed. The pressure-like $W_{+}$has a rapidly varying feature near the walls, but the feature is free of multiple inflections as in the plane wave mask case. This simpler structure of the wall pressure anomaly for the sine basis method apparently leads to better stability characteristics of both explicit and semiimplicit field updates, and the use of larger time steps that dramatically accelerates convergence as evidenced in Fig. 2. The improved performance of the SIS scheme with the sine basis is particularly notable. While plane wave simulations of mask-confined block copolymer melts are difficult to converge below an error of $O\left(10^{-6}\right)$, the sine basis method coupled with SIS field relaxation allows SCFT simulations to be carried out to machine precision within a few thousand iterations. 


\section{AB Diblock Copolymer Melt in a Square Well}

In order to examine how square confinement affects block copolymer thin film morphologies, two-dimensional SCFT simulations of AB diblock copolymer melts were performed. Throughout all of the simulations, the volume fraction of A segments on each diblock, $f$, was fixed at 0.7 and the A- or B-attractive wall condition was specified by setting $\chi_{w} N$ to 17 or -17 , respectively. A spectral collocation approach was followed using a two-dimensional sine basis coupled with SIS field relaxation as described above. The simulations were initialized with random initial field configurations and were annealed from $\chi N=12$ to $\chi N=17$ [above the order-disorder transition (ODT)] in steps of 0.25 . Each $\chi N$ step was simulated for 500 iterations, resulting in a total run of 10,000 iterations. We note that our field relaxation scheme does not produce mass transport that coincides with either diffusive dynamics or hydrodynamic flow. Nonetheless, we expect our slow annealing procedure, which maintains a quasi-static equilibrium at each $\chi$ or $T$, should faithfully reproduce experimental observations of microphase evolution under slow thermal annealing protocols.

As shown in a previous SCFT study of AB block copolymer thin films confined in a hexagonal well, ${ }^{21}$ low-amplitude composition oscillations radiating from the boundary into the bulk were shown to induce microphase ordering. Bosse et al. ${ }^{21}$ further identified commensurate windows of confinement wall side length that yield a defect-free hexagonal array of cylindrical microdomains. In our simulations of square confined block copolymer films, we similarly observed commensurate windows of side length $L$ that generate defect-free ordering. While Bosse et al. focused on obtaining large-area defect-free hexagonal ordering, which is the favorable phase in the bulk, here we are concerned with obtaining tetragonal lattices of cylinders, which are not possible in the bulk. In particular, our focus is the competition between confinement-induced square ordering and the bulk tendency for hexagonal ordering.

Square ordering is not achievable in an unconfined bulk system, but by using a square well, the confined geometry creates a competition between the natural tendency toward forming hexagonal lattices of $\mathrm{B}$ cylinders and the propagating tetragonal symmetry of the confinement throughout the system. We have observed that a properly sized square confinement can create a 
perfect square lattice throughout the annealing stage, even though it is thermodynamically favorable to undergo hexagonal ordering in the bulk. For small sized systems, the well edge-induced composition waves are dominant over the entire domain; thus it is possible for a square lattice to be more stable than a hexagonal lattice. As an example, we were able to stabilize a square $(3 \times 3)$ lattice of 9 B-cylinders from an $\mathrm{AB}$ diblock copolymer thin film confined in a A-attractive square well with a side length of $L=10 R_{g}$. However, when the system size increases beyond $L \approx 13 R_{g}$, we observed that tetragonal ordering developed through the annealing process is metastable or becomes unstable, and does not persist under continued annealing. The ultimate morphology in this case is a defective, "twisted hexagonal" phase that will be discussed further below.

Figure 3 shows representative composition profiles (the color bar indicates the intensity of A monomer volume fraction) after various numbers of iterations as a diblock copolymer melt with $L=14 R_{g}$ and an A-attractive wall $\left(\chi_{w} N=17\right)$ is annealed throughout a simulation. The background around each square well is set to black to emphasize the confined area. However, the simulation domain corresponding to the square well excludes the black annular region. In our masking technique, the total polymer segment density decays to zero at the boundary, which is reflected in a tiny depletion layer of A segments around the boundary at each representative density plot.

The panels in Fig. 3 correspond to the $4000^{\text {th }}(\chi N=13.75), 6000^{\text {th }}(\chi N=$ $15.75), 10000^{t h}(\chi N=17)$ and $30000^{t h}(\chi N=17)$ iteration. We note that the tetragonal ordering starts to develop at temperatures above the bulk ODT $(\chi N=14)$. Although the simulation forms a perfect square lattice during the intermediate stage of the annealing process, further relaxation shows that the tetragonal structure, while free of defects, is metastable. Immediately after the annealing stage ends (third panel in Fig. 3), we observe tetragonal order with an associated SCFT error, the $l_{1}$ norm of the field-gradient of the Hamiltonian, at machine precision, indicating that the tetragonal lattice is at least a long-lived structure on the free-energy landscape. However, as the simulation progresses at constant $\chi N$, the $\mathrm{B}$ cylinders slowly migrate and twist as shown in the right most panel. We have confirmed that this "twisted hexagonal" morphology has a lower free energy than the perfect square lattice at any square size $L$ within the commensurate window that encloses 16 $\mathrm{B}$ cylinders $(4 \times 4$ tetragonal lattice). The implication is that (for this range of $L$ ) the favorable surface free energy of the tetragonal state caused by the interaction with the wall cannot compensate for the unfavorable bulk stress of 
the highly stretched coronal A chains necessary to fill the interstitial sites of a square lattice. Nonetheless, the free energy difference (per chain) between the square and twisted hexagonal structures is very small, i.e. $O\left(10^{-2}\right)$. As a result, it is possible to slightly modify the formulation of our system in order to stabilize the square lattice relative to the twisted hexagonal phase.

\section{AB Diblock Copolymer/A Homopolymer Blend in a Square Well}

One approach to stabilizing square lattices is to reduce the bulk stress associated with the highly stretched A segments in the block copolymer. An additive, such as an A (majority block) homopolymer, can act in this regard by occupying regions in the microstructure that otherwise would be filled by highly stretched coronal A blocks of the copolymers. ${ }^{25,31}$ To explore this strategy in the present context of square confinement, we have conducted SCFT simulations of AB diblock copolymer/A homopolymer blends in a square well, varying both the molecular weight and the volume fraction of the A homopolymer additive. For this purpose, we define $\alpha$ to be the molecular weight ratio of homopolymer to diblock, $\alpha=N_{A h} / N$, and $V_{A h}$ as the volume fraction of $\mathrm{A}$ homopolymer contained in the blend. The SCFT simulations were started from random initial field configurations and the simulations were annealed stepwise in the same way as in the previous AB diblock copolymer system. In addition to the annealing protocol, most of the simulation parameters, such as $f$ and $\chi_{w} N$, were fixed at the same values as for diblocks in the previous section. Our simulation results show that the addition of homopolymer to the system can indeed stabilize the square configuration. Moreover, we have observed that the A homopolymer additive shifts the $4 \times 4$ commensurate window of side length $L$ to larger values depending on $\alpha$ and $V_{A h}$, similar to the result of Bosse et al. ${ }^{21}$

By computational exploration of the three-dimensional parameter space $\left\{\alpha, V_{A h}, L\right\}$, we have observed several interesting types of microdomain ordering and have established optimal parameter sets necessary to thermodynamically stabilize defect-free tetragonal ordering in square-confined binary blends of $\mathrm{AB}$ diblock copolymer with A homopolymer. 


\subsection{Observed Phases}

Before discussing the optimal parameters necessary to stabilize square lattices, we first introduce the different microdomain structures observed for block copolymer/homopolymer blends confined in a square well. For each stable or metastable structure, which will be referred to as a "phase," we discuss not only how the phase evolves throughout the annealing stage, but also how the A homopolymer acts to stabilize or destabilize it.

When low molecular weight A homopolymers are added at low concentrations to $\mathrm{AB}$ diblocks, twisted hexagonal ordering (HEX) is favored as in the pure AB diblock case. The left three panels of Fig. 4(a) show how the total A segment composition, i.e. the sum of the A segment densities contributed by the A homopolymers and the A blocks of the copolymers, evolve during an SCFT simulation for a system with $\alpha / f=2.5$ and $V_{A h}=0.17$ confined in a $21 R_{g} \times 21 R_{g}$ square well with B-wetting conditions on the perimeter. The rightmost panel of the figure shows the A homopolymer segment composition at the equilibrium state. We note that in exploring homopolymer molecular weight effects, it is natural to control the parameter $\alpha / f$, which is the ratio of the molecular weight of the A homopolymer to the molecular weight of the $A$ block of the AB diblock. The degree to which A homopolymer will penetrate the A coronas of the cylindrical microstructure and thereby establish "wet brush" or "dry brush" conditions is evidently controlled by this molecular weight ratio.

It is shown in the left three panels of Fig. 4(a), as in the case of a squareconfined diblock melt, that B-wetting walls promote tetragonal ordering of a square-confined blend upon annealing, which subsequently distorts into the twisted hexagonal phase. It is also evident that the transient tetragonal order initiates from the corners and edges of the square well. The rightmost panel shows that the A homopolymer density is relatively uniform outside the $\mathrm{B}$ cylinders, which indicates that the A homopolymer is highly miscible with the A block micelle coronas at this segregation strength and $\alpha / f=2.5$. Homopolymers of this length and shorter are unwilling to pay the translational entropy cost of partitioning into the interstitial sites and thereby stabilizing the tetragonal phase. Nevertheless, the free energy difference between the square and twisted hexagonal configurations is reduced compared to the neat AB diblock case.

The addition of greater amounts of longer A homopolymer into the system decreases the free energy of the square configuration such that it is energet- 
ically more favorable than the twisted hexagonal configuration. Fig. 4(b) shows that tetragonal order (SQR) is developed through the annealing process and persists when A homopolymer with $\alpha / f=2.5$ and at volume fraction $V_{A h}=0.23$ is blended with block copolymer into the B attractive $23 R_{g} \times 23 R_{g}$ square well. The rightmost panel in Fig. 4(b) shows how the A homopolymer stabilizes the tetragonal phase by preferentially locating in the interstitial sites, thus eliminating the need of the A blocks of the copolymer to stretch into those regions.

Macroscopic phase separation (MAC I) occurs when an excess amount of short to moderate length A homopolymer, or a small amount of long A homopolymer are added. With an excess amount of short A homopolymer, the A-homopolymer behaves like a solvent and easily mixes to swell the block copolymers. Thus, as shown in Fig. 4(c), the A homopolymer decreases the degree of segregation between A and B segments: ordering develops at increased $\chi N$. Hence the final morphology is similar to that observed in a system with longer A homopolymer at $\chi N$ slightly below the ODT as shown in the left most panels in Figs. 4(a) (HEX) and 4(b)(SQR). The system ends up in Fig. 4(c) with the disordered state in the center of the template, where block copolymer and A homopolymer mix together and each component has uniform density profile, while the block copolymer preferentially segregates to the walls and generates local microdomain order. Strictly speaking, this is neither a disordered state nor macroscopic phase separation, but a pre-transitional wetting profile. However, because this phase smoothly transitions into the macroscopic phase separation pattern shown in Fig. 4(d) as the A homopolymer length is increased, we shall refer to this state as macroscopically phase separated (MAC I).

For very long A homopolymer additives, depending on the wall wetting conditions, the system macrophase separates in two ways. For a B-attractive wall, the A homopolymer localizes in the center of the square well rather than next to the wall and it generates the MAC I pattern as shown in Fig. 4(c). In this phase, i.e. Fig. 4(d), we observe a highly concentrated A homopolymer density in the middle while most of the block copolymer locates next to the wall where we observe cylinders of B. Unlike the B-attractive wall, an A-attractive wall sequesters long A homopolymers to reside near the wall. As shown in Fig. 4(e), long A homopolymers are attracted to the wall while the block copolymer localizes and microphase separates in the center of the square domain (MAC II). This configuration, in which A homopolymer segments rather than A block segments are located at the wall, is apparently 
preferred because it relieves excess chain stretching that copolymers at the wall would otherwise incur.

A particularly interesting aspect of the early stage ordering to the MAC I phase, Figs. 4(c) and 4(d), is that cylinders form first in the four corners of the square and then subsequently spawn more cylinders to fill in the faces. This corner phenomenon is present to some extent in all B-wetting simulations, but is most pronounced under blend conditions that lead to the MAC I phase. The observation could provide a strategy to create isolated cylindrical "posts" in the corners of a square, rather than a dense tetragonal lattice, by rapidly quenching the melt after annealing slightly above the ODT.

As an intermediate structure between MAC I and SQR, there exists another type of macroscopic phase separation (MAC III), which consists of a single B cylinder in the middle of the square, a ring of B cylinders along the walls of the square well, and homopolymer preferentially located in the annular region between as shown in Fig. 4(f). The MAC III phase is also observed by increasing the segregation strength from phase MAC I for short A homopolymers (Fig. 4(c)).

Figures 4(a) through 4(f) summarize the primary structures that have been observed in a large number of SCFT simulations with A-wetting and Bwetting walls, and for square cells in the $4 \times 4$ commensurate window having sizes in the range $17 R_{g} \leq L \leq 23 R_{g}$ and $22 R_{g} \leq L \leq 24 R_{g}$ for A-wetting and B-wetting walls, respectively. A number of defective variants of these phases were also observed in a few simulations, although these are believed to be metastable relative to the primary structures identified. We also have tried to obtain larger sized square lattices such as a $5 \times 5$. Unfortunately, we were not able to achieve such larger structures, even with the addition of homopolymer, presumably because the wall-induced tetragonal order is a surface energetic effect that is overwhelmed for large wells by the bulk energetic preference for hexagonal order. ${ }^{32,33}$ Nonetheless, a $4 \times 4$ lattice constitutes a square well of 50-100 $\mathrm{nm}$ on a side, which is easily accessed by state-of-the-art lithography, and allows for an effective four to six fold reduction in feature size below the optical limit.

\subsection{Wall Wetting Conditions}

Beyond confirming that both A-wetting and B-wetting conditions on the square walls can assist in promoting tetragonal ordering, we also conducted a series of simulations to quantify the relative influence of the two wall treat- 
ments on the width and location of the $4 \times 4$ commensurate stability window for the square phase.

We show in Fig. 5 the observed phase morphologies as a function of the homopolymer volume fraction $V_{A h}$ for both A-wetting and B-wetting walls, at a fixed length fraction of A homopolymer $(\alpha / f=3.0)$. Since the B-wetting wall attracts the $\mathrm{B}$ segments in the block copolymer, a mono-lamellar coating is formed along the wall. Because of this coating, the size of the square well needed for a strain-free $4 \times 4 \mathrm{~B}$-cylinder lattice is larger than that needed for an A-wetting wall as shown in Fig. 5. In addition, as more A homopolymer is added, the swelling of the A corona regions of the micelles implies that an even larger square well is needed to sustain the $4 \times 4$ lattice; this accounts for the increase of the square well from left to right in Fig. 5. Twisted hexagonal ordering is observed for small amounts of A homopolymer additive as shown for $V_{A h}=0.18$. For large amounts of A homopolymer, macroscopic phase separation is observed, while for intermediate values of $V_{A h}$ regions of tetragonal ordering are obtained for both A- and B-attractive walls. We observe a window of A-homopolymer volume fraction $V_{A h}$ that produces stable tetragonal ordering, and correspondingly, a "commensurate window" of confinement wall side length $L$ that leads to the SQR phase. Thus, by tuning the volume fraction $V_{A h}$ (or length fraction $\alpha$ ) of the A homopolymer additive, or the side length of the square well, we can generate $4 \times 4$ tetragonal lattices of $\mathrm{B}$ cylinders with different domain spacings. This continuous tunability of tetragonal feature spacing should enhance the practical applicability of $\mathrm{AB} / \mathrm{A}$ blends in block copolymer lithography.

As explained in the previous section, the mechanism of stabilizing microscopic tetragonal order requires that the A homopolymers concentrate in the interstitial sites. Thus, it is expected that the B-wetting wall would be more effective than the A-wetting wall at situating the A-homopolymers in the interstitial sites rather than near the wall, and consequently aid in stabilizing the tetragonal phase. However, the simulation results shown in Fig. 5 demonstrate that the A-wetting well condition provides a wider stability window for the square phase. While the transition from twisted hexagonal ordering to tetragonal ordering occurs around the same volume fraction of A homopolymer for both A- and B-wetting wall conditions, macroscopic phase separation occurs for the B-wetting wall at much smaller $V_{A h}$ than for the A-wetting wall. Fig. 6 shows the A homopolymer segment volume fraction at $V_{A h}=0.25$ for a B-wetting wall (a) and an A-wetting wall (b). Evidently, the B-wetting wall condition prevents the uniform distribution of the A ho- 
mopolymer over the interstitial sites; instead, the homopolymer has a higher density in the middle of the well rather than near the wall. This can be understood because, at a B attractive wall, the block polymer localizes there and forms a planar dense brush of A blocks that repels the A homopolymer. In contrast, for an A attractive wall, no dense copolymer brush is built up that limits access of the homopolymer to the wall region. Since macroscopic phase separation occurs when the A homopolymer segregates to the center of the well, it is understandable that B-wetting walls assist in the development of macroscopic phase separation. Based on this result, namely that A-wetting walls perform better in promoting tetragonal ordering, we will subsequently restrict our attention to A-wetting conditions. However, it is notable that in certain systems, A-wetting walls will be problematic because excessively strong attractions can pin components at the well edges and dramatically increase equilibration times. ${ }^{2,34}$

\section{Phase Diagram Based on Annealed Simu- lation and Free Energy Tracking}

In the previous section, it was demonstrated that persistent, stable tetragonal ordering could be obtained from SCFT simulations that were started from random initial field configurations and annealed while gradually increasing the segregation force. Since the confined system could structure in many possible ways that were not possible to predict a priori, such annealed/random initial condition simulations were a necessary first step in order to establish a set of candidate phases that could be considered in a phase diagram. However, the final morphology observed in an annealed simulation may not necessarily be in thermodynamic equilibrium, but instead be in a kinetically-arrested metastable state. Thus, in order to obtain a more precise and comprehensive phase diagram, we have determined the lowest free energy (stable) state as a function of the length and volume fraction of the A homopolymer additive by comparing the free energies of the candidate phases. This free energy tracking proves the thermodynamic stability of the square ordering and also allows us to obtain a more reliable phase diagram that can be used as a guide for practical block copolymer lithography.

In performing free energy tracking, we seeded SCFT simulations with the field configurations of candidate phases obtained from annealed/random 
initial field simulations, and discussed in the context of Fig. 4. After relaxing the fields to convergence for a specified (new) set of parameters, the free energy associated with each phase is calculated. A phase boundary is then calculated by forming the locus of the zero-crossing points of the free energy difference between two neighboring phases. In the following sections we introduce phase diagrams for $\mathrm{AB}+\mathrm{A}$ thin film blends confined in an A-wetting square well, where results from both the annealed simulations and the free energy tracking method are included.

\subsection{Phase Diagram at Fixed $L$}

The phase diagram for an $\mathrm{AB}+\mathrm{A}$ thin film confined in a $17 R_{g} \times 17 R_{g}$ sized A-wetting square well, as a function of $\alpha / f$ and $V_{A h}$, is shown in Fig. 7. Each phase observed from an annealed simulation initialized with a random field configurations is marked as a symbol with the corresponding marker, while boundaries obtained from free energy tracking are drawn as continuous curves. Three boundaries were deduced from free energy tracking: between HEX and SQR, SQR and MAC III, and between MAC I and MAC III.

We show in Figure 7 that the phase boundary between hexagonal (HEX) and square (SQR) ordering is consistently predicted using both the annealed simulations and those employing free energy tracking. Nonetheless, we observed that vertical (constant $\alpha$ ) and horizontal (constant $V_{A h}$ ) free energy tracking produced a slightly different boundary; hence the free energy derived boundary is indicated with a thick line. A possible explanation for the difficulty in obtaining accurate predictions for this phase boundary via free energy tracking relates to the fact that the hexagonal phase is actually a defective phase that is twisted from perfect square ordering due to the bulk tendency towards hexagonal ordering. Furthermore as the length and volume of the A homopolymer additive approaches the condition of square ordering, the amplitude of the twist decreases in a nearly continuous fashion and the free energy difference between the hexagonal and square phases decreases in concert. The associated loss of driving force to relax the fields near this apparently continuous transition accounts for the numerical difficulty in accurately discerning the phase boundary.

With regard to the boundary between macrophase separation (MAC III) and square (SQR) packing, free energy tracking leads to a phase boundary that is considerably higher in homopolymer volume fraction than the one obtained through annealed simulations. In the annealed simulations, the 
early wall-induced ordering by the A-attractive walls leads the system into a configuration where the B-cylinders are situated around the wall but not in the middle, which is close to a configuration of macroscopic phase separation (MAC I or III). During the subsequent evolution of ordering, the system is therefore easily trapped in a metastable macroscopic configuration instead of more stable square ordering due to its kinetic path. There exists a band of MAC III between the SQR and MAC I, which is also observed in the annealed simulations. For $\alpha / f>5$, which corresponds to the addition of very long A homopolymer, both phase boundaries terminate and the second type of macroscopic phase separation (MAC II, Fig. 4(e)) becomes stable. Since we are primarily interested in finding parameters that enhance tetragonal ordering, we did not further explore the region where MAC II is stable.

A similar phase diagram for $18 R_{g} \times 18 R_{g}$ square well confinement is drawn and shown in Fig. 8. Qualitatively, the shapes of the upper and lower boundaries of the square phase are similar to the boundaries obtained in Fig. 7, but the overall square ordering region is extended to larger volume fractions. Another difference is that the twisted hexagonal phase is no longer observed, but is replaced by the defective square phase (d-SQR) with a point defect (extra cylinder) in the center.

\subsection{Phase Diagram at Fixed $\alpha$}

In this section, the phase diagram is shown by tracking the free energy density at fixed A homopolymer length fraction, $\alpha / f=3.0$, and expressing the phase behavior as a function of $V_{A h}$ and $L$. For a large square well (upper region in Fig. 9), the incommensurate confinement effect is large, so the defective square phase (d-SQR) with an additional cylinder in the middle of the well, is stabilized. Below this region, tetragonal ordering (SQR), hexagonal ordering (HEX), and macroscopic phase separation (MAC III and MAC I) are sequentially observed as stable states. Between the d-SQR and MAC III phases, hexagonal ordering intervenes at low volume fractions of homopolymer, while the tetragonal phase (SQR) is intermediate with sufficient amounts of A homopolymer additive. The left side of the phase diagram, corresponding to very small amounts of A homopolymer, shows a vertical phase sequence similar to a neat $\mathrm{AB}$ diblock copolymer: tetragonal ordering is not stabilized and we observe a large window of $L$ for hexagonal ordering. However, the HEX region narrows and finally disappears around $V_{A h}=0.3$ and $L=16 R_{g}$. For larger $V_{A h}$, the MAC III and I phases are observed after 
SQR with decreasing confinement size. Location of the precise boundary between hexagonal and tetragonal ordering is again difficult due to the apparent continuous nature of the transition, so this boundary should be viewed as an approximate guide. The two horizontal cuts through the phase diagram at $L=17$ and 18 correspond to the vertical cuts at $\alpha / f=3.0$ in the previous two phase diagrams at constant $L$. For $L=17 R_{g}$, hexagonal, tetragonal and macrophase ordering type III and I are successively observed with increasing $V_{A h}$, while for $L=18$ the defective square phase is observed instead of the twisted hexagonal phase at small $V_{A h}$.

\section{Influence of Line Edge Roughness}

In the practical implementation of graphoepitaxial block copolymer lithography, conventional top-down optical lithography would be used to define confining wells for the block copolymer assembly, such as the square wells considered here. However, the perimeters of such wells cannot be defined and developed with sub $10 \mathrm{~nm}$ resolution, resulting in wells with significant line edge roughness on the block copolymer assembly scale. ${ }^{35}$ To investigate the effect of line edge roughness on the self-assembly of confined block copolymers, we have used SCFT simulations to study the influence of boundary perturbations of the square well on feature defect populations. Our primary focus is on establishing the robustness of the SQR phase to boundary perturbations of varying wavelength and amplitude.

To implement arbitrary boundary perturbations in SCFT simulations, it is most convenient to return to the masking method, embedding the mask in a larger square domain subject to periodic boundary conditions and employing collocation with plane waves. Because the mask can be of arbitrary shape, a wide variety of wells can be readily generated, such as those having rounded corners or wavy walls. As discussed above, the masking technique leads to some numerical difficulties, but is nonetheless suitable for the investigation at hand.

We show in Figure 10(a), (b) and (c) representative composition profiles for an $\mathrm{AB}$ diblock/A homopolymer blend thin film confined in a $16 R_{g} \times 16 R_{g}$ A-wetting square well with three examples of line edge roughness: rounded corners, high frequency perturbations, and low frequency perturbations, respectively. The homopolymer length and volume fraction are set at values, $\alpha / f=2.5$ and $V_{A h}=0.25$, respectively, that lead to perfect tetragonal 
order when there is no line edge roughness. The leftmost panel shows that rounded corners, which are frequently observed in lithographically fabricated square wells, ${ }^{36,37}$ do not disrupt ordering of the square phase. In the second panel, where high spatial frequency but low amplitude boundary perturbations have been imposed, we observe robust and defect-free tetragonal order. Apparently the high wave number $(k)$ boundary perturbations are not translated into wall-induced composition waves in the block copolymer medium of comparable frequency. A similar damping phenomenon is well known for the equilibrium response of bulk block copolymers subjected to a high $k$ chemical potential field.$^{38}$ Boundary perturbations are more effective at disrupting microdomain order, however, as the amplitude and wave length of the roughness is increased. For example, the rightmost panel of Fig. 10 shows that a moderate amplitude, long wavelength modulation of the square walls is capable of destroying the SQR phase.

To expand on these qualitative results, we have performed a more systematic SCFT study of line edge roughness effects by superimposing on a square mask a harmonic boundary perturbation of the form $A \cdot \cos [2 \pi(x-\Delta) /(\lambda L)]$, where $x$ is a square boundary coordinate with origin in the center of each segment of length $L=16 R_{g}$, and $\lambda, A$, and $\Delta$ represent the wavelength, amplitude, and phase shift of the perturbation, respectively. The role of the parameters $\lambda, A$, and $\Delta$ on the robustness of tetragonal ordering was investigated by conducting a large number of SCFT simulations, each started from a random initial condition, and collecting statistical information about the quality of the resulting block copolymer assembly.

As an indicator of how close the obtained microdomain ordering is to perfect tetragonal ordering, we have utilized an order parameter $\Psi$ that is a square sum of the difference between the pattern and a reference perfect tetragonal pattern over all grid points:

$$
\Psi=\sum_{i}^{M}\left[\phi_{B}(i)-\phi_{B, r e f}(i)\right]^{2}
$$

where $i$ indexes the grid points, $M$ is the total number of grid points, and $\phi_{B}$ and $\phi_{B, \text { ref }}$ represent the densities of $\mathrm{B}$ segments (minor component in cylinder cores) for the simulated system and a reference system with perfect tetragonal ordering, respectively. This order parameter therefore provides a measure of the deviation of $\mathrm{B}$ segment density from a perfect tetragonal pattern. 
We show in Figure 11 intensity maps of the order parameter in the spatial frequency $(1 / \lambda)$ and amplitude $(A)$ domain for different phase shifts $\Delta$. In Figs. 11(b) and (c), the cosine wave has been shifted from the origin by an amount of 0.5 and $1.0 R_{g}$, respectively. In this way, asymmetric perturbations have been imposed on the wall. How $\Delta$ imposes the asymmetries on the wall is shown in Fig. 12. In the intensity maps, blue regions indicate a high susceptibility to tetragonal ordering with a small order parameter, while red denotes a defective phase of large order parameter. In the low frequency limit, we observe the largest distortion of tetragonal ordering. As we increase the perturbation asymmetry by increasing the phase shift, a wider region of defective ordering is observed. Valleys in the low frequency region correspond to the case when the microdomain period is a multiple of the perturbation wavelength and thus wall modulation has little influence on the tetragonal structure. Although the asymmetrical case of slowly varying line edge roughness can cause significant disruption of ordering, such wall defects rarely occur in conventional lithography. On the other hand, when the amplitude of perturbation is small, tetragonal ordering is observed even for highly-asymmetric, low-frequency wall perturbations. It is also shown in Figure 11 that the SQR phase is robust to high frequency perturbations of the wall, regardless of the asymmetry and the amplitude. Since all confinement wells created by top-down lithography realistically exhibit some roughness, our results provide a simple framework for understanding when line edge roughness will create practical impediments to block copolymer lithography.

\section{Summary and Conclusions}

The present paper provides an example of how lateral confinement of thin block copolymer films can produce microphase structures that are not present in bulk, unconfined systems. While this concept is not new, we have exploited it in a modern context to create square lattices of "standing up" cylinders that are highly desirable in the emerging field of block copolymer lithography.

Specifically, we conducted two-dimensional SCFT simulations to determine optimal conditions by which lateral confinement (graphoepitaxy) can promote perfect long-range tetragonal order of cylindrical block copolymer features. For an AB diblock copolymer thin film system confined to a square well, we have confirmed that A-attractive and B-attractive walls assist in generating composition waves from the walls that template tetragonal mi- 
crodomain ordering throughout the domain. For square wells of a size commensurate with a $3 \times 3$ square lattice of 9 cylinders, we have found that the tetragonal phase can be thermodynamically stable. However, for square wells compatible with a $4 \times 4$ square lattice of 16 cylinders, the tetragonal phase appears as a transient structure that is metastable relative to a twisted hexagonal morphology. For square wells of this size and larger, due to the bulk thermodynamic preference of packing cylinders hexagonally, it is not possible to achieve globally stable tetragonal lattices in a pure AB diblock melt film. However, by adding a suitable A homopolymer additive to the system that can relieve stretching of the coronal A blocks, we were able to stabilize $4 \times 4$ square lattices composed of B cylinders. Depending on the length and volume fraction of the homopolymer additive, a number of different phase morphologies were identified. Phase diagrams were constructed based on the SCFT results that suggest how to tune homopolymer molecular weight, volume fraction, and side length of the square well in order to achieve robust, defect-free square lattices of cylinders.

We also performed numerical investigations of the role of line edge roughness at the periphery of the square well on defect populations. Through a series of SCFT simulations, the combined effect of the amplitude, wavelength, and asymmetry of harmonic boundary perturbations on the establishment of tetragonal order was established. Our findings of a strong robustness of tetragonal order to line edge roughness support the practical feasibility of block copolymer lithography coupled with graphoepitaxy.

It is notable that our results were obtained by assuming homogeneity in the polymer film normal to the substrate; this assumption of vertically oriented cylinders is valid only when the top and bottom surfaces do not have selective interactions with either the A or B polymer segments. Such a situation, i.e. where the top and bottom surfaces are neutral but where the side walls of the square are selective, can in principle be experimentally realized by depositing a selective wall on top of the neutral substrate using the lift-off technique, or by employing two substrate layers (a top selective layer and a bottom neutral layer) and etching square wells into only the top layer. In practice, it may prove difficult to exactly neutralize the underlying substrate and the top surface of the film. To fully describe such systems, it will be necessary to conduct three-dimensional (3D) SCFT simulations. Nonetheless, we expect that the current 2D model and results, although limited, will provide useful guidance for experimental designs. 


\section{Acknowledgement.}

SH would like to thank T. L. Chantawansri, A. J. Onikoyi and N. A. Lynd for useful discussions. SH, CJGC and GHF derived partial support from NSF Grant No. DMR-0603710 and the MARCO Center on Functional Engineered Nano Architectonics (FENA). EJK was partially supported by NSF Grant No. DMR-0704539. This work made use of MRL Central Facilities supported by the MRSEC Program of the National Science Foundation under award No. DMR-0520415.

\section{Appendix}

In this Appendix we summarize the blend model and relevant equations and numerical methods that were used in the present SCFT simulation study. The notation, formalism, and techniques are similar to those discussed in a previous paper by Bosse et al. ${ }^{21}$ so we refer the interested reader to that publication for further details.

\section{SCFT Model Equations: AB Diblock Copolymer/A Ho- mopolymer Blend in a Square Well}

In our model system we consider $n_{d}$ and $n_{A h}$ monodisperse $\mathrm{AB}$ diblock copolymers and A homopolymers, respectively, in a volume $V$, along with $n_{w}$ wall particles. The wall particles are introduced in order to implement a lateral confinement wall that interacts with polymer segments $\mathrm{A}$ and $\mathrm{B}$ by means of Flory-type interaction parameters $\chi_{w A}$ and $\chi_{w B}$, respectively. The index of polymerization of the diblock copolymer and homopolymer are $N$ and $N_{A h}$, respectively. Moreover, the length fraction of the A homopolymer relative to the block copolymer is defined as $\alpha=N_{A h} / N$. Since we assume that the statistical lengths and volumes of each segment are equal for all the species, $n_{d}$ and $n_{A h}$ are related by the A homopolymer volume fraction,

$$
V_{A h}=\frac{n_{A h} N_{A h}}{n_{d} N+n_{A h} N_{A h}}=\frac{\alpha n_{A h}}{n_{d}+\alpha n_{A h}}
$$

While the microscopic segment density $\hat{\rho}$ is a function of the instantaneous chain configurations, the wall density $\rho_{w}(\mathbf{r})$ is a specified smooth function. $\rho_{w}(\mathbf{r})$ is set to zero in the interior of the confined well and $\rho_{0}=\left(n_{d} N+n_{w}+\right.$ 
$\left.n_{A h} N_{A h}\right) / V$, the average total segment density, in the exterior. To transition between the two values at the wall boundaries we use a tanh function, $\rho_{0}\left[1-\tanh \left(\frac{d_{\perp}(x)}{\delta}\right)\right]$, which incorporates a narrow and smooth interfacial region. $d_{\perp}(x)$ is defined as the distance from the point $x$ to the nearest edge of the boundary of the square well and $\delta \approx 0.2$ is chosen so that $\rho_{w}$ is dropped to $O\left(10^{-2}\right)$ at $d_{\perp}(x)=1 R_{g}$. This "diffuse interface" approach serves to reduce numerical instability associated with sharp interfaces, while providing a physically realistic description of a bound system with a sharp interface.

Melt incompressibility is enforced by the following constraint on the microscopic polymer segment densities:

$$
\hat{\rho}_{A c}(\mathbf{r})+\hat{\rho}_{B c}(\mathbf{r})+\hat{\rho}_{A h}(\mathbf{r})+\rho_{w}(\mathbf{r})=\rho_{0}
$$

where the subscripts $c$ and $h$ represent the copolymer and homopolymer, respectively. This constraint, coupled with the specification of the wall density field, effectively restricts the polymer segments to the interior of the confinement well. ${ }^{21,29,39}$ The canonical partition function is given by:

$$
\begin{aligned}
Z= & \int \prod_{k=1}^{n_{d}} \mathcal{D} r_{k} \prod_{l=1}^{n_{A h}} \mathcal{D} r_{A h, l}\left[\delta\left(\hat{\rho}_{A c}+\hat{\rho}_{B c}+\hat{\rho}_{A h}+\rho_{w}-\rho_{0}\right) \times\right. \\
& \left.\exp \left(-U_{0}\left[\left\{r_{k}, r_{A h, l}\right\}\right]-U_{I}\left[\left\{r_{k}, r_{A h, l}\right\}\right]\right)\right]
\end{aligned}
$$

where $U_{0}$ and $U_{I}$ are the harmonic stretching energy of the Gaussian chain and the segment interaction energy, respectively, and are denoted by:

$$
\begin{gathered}
U_{0}\left[\left\{r_{k}, r_{A h, l}\right\}\right]=\frac{1}{4 R_{g}^{2}}\left(\sum_{k=1}^{n_{d}} \int_{0}^{1} d s\left|\frac{d r_{k}(s)}{d s}\right|^{2}+\sum_{l=1}^{n_{A h}} \int_{0}^{\alpha} d s\left|\frac{d r_{A h, l}(s)}{d s}\right|^{2}\right) \\
U_{I}\left[\left\{r_{k}, r_{A h, l}\right\}\right]=\frac{1}{\rho_{0}} \int_{V} d \mathbf{r}\left[\chi \hat{\rho}_{A}(\mathbf{r}) \hat{\rho}_{B}(\mathbf{r})+\chi_{w A} \rho_{w}(\mathbf{r}) \hat{\rho}_{A}(\mathbf{r})+\chi_{w B} \rho_{w}(\mathbf{r}) \hat{\rho}_{B}(\mathbf{r})\right]
\end{gathered}
$$

The objects $r_{k}(s)$ and $r_{A h, l}(s)$ denote space curves representing the configuration of a Gaussian chain of copolymer and an A homopolymer with chain indexes $k$ and $l$. Furthermore, the total microscopic densities of $\mathrm{A}$ and $\mathrm{B}$ segments appearing in $U_{I}$ are defined by

$$
\hat{\rho}_{A}(\mathbf{r})=\hat{\rho}_{A c}(\mathbf{r})+\hat{\rho}_{A h}(\mathbf{r}), \quad \hat{\rho}_{B}(\mathbf{r})=\hat{\rho}_{B c}(\mathbf{r})
$$


This coarse-grained particle model is converted into a field theory as described in the previous paper. ${ }^{21}$ First, instead of the microscopic segment density of each species, two microscopic densities defined as

$$
\hat{\rho}_{ \pm}=\hat{\rho}_{A} \pm \hat{\rho}_{B}
$$

are introduced. After enforcing the incompressibility constraint and imposing a constant shift in the energy, Hubbard-Statonovich transformations are performed to convert the model into a field theory. An expression for the canonical partition function is obtained in terms of a functional integral with a statistical weight over two conjugate potential fields, $W_{+}, W_{-}$

$$
Z=\int \mathcal{D} W_{+} \mathcal{D} W_{-} \exp \left(-H\left[W_{+}, W_{-}\right]\right)
$$

where the effective Hamiltonian has the following form:

$$
\begin{aligned}
H\left[W_{+}, W_{-}\right]=C & \int_{V} d \mathbf{x}\left[\frac{1}{\chi N} W_{-}^{2}(\mathbf{x})-\imath \phi(\mathbf{x}) W_{+}(\mathbf{x})-\frac{2 \chi_{w} N}{\chi N} \phi_{w}(\mathbf{x}) W_{-}(\mathbf{x})\right](10) \\
& -C\left(1-V_{A h}\right) \bar{\phi} V \ln \mathcal{Q}\left[W_{A}, W_{B}\right]-C \frac{V_{A h} \bar{\phi} V}{\alpha} \ln \mathcal{Q}_{A h}\left[W_{A}\right]
\end{aligned}
$$

with $C=\rho_{0} R_{g}^{3} / N$, normalized wall density $\phi_{w}=\rho_{w}(\mathbf{x}) / \rho_{0}$ and total polymer segment density $\phi(\mathbf{x})=\rho(\mathbf{x}) / \rho_{0}=1-\rho_{w}(\mathbf{x}) / \rho_{0}$, and its spatial average $\bar{\phi}$. $\chi_{w}$ is defined as $-\left(\chi_{w A}-\chi_{w B}\right) / 2$, so that it is positive for a A-wetting wall and negative for a B-wetting wall. Potential fields $W_{A}$ and $W_{B}$ that act on $\mathrm{A}$ and $\mathrm{B}$ segments, respectively, are defined as $W_{A}=\imath W_{+}-W_{-}$ and $W_{B}=\imath W_{+}+W_{-}$, where $W_{+}$is an imaginary pressure-like field that enforces incompressibility and $W_{-}$is a real-valued exchange potential field. $\mathcal{Q}\left[W_{A}, W_{B}\right]$ and $\mathcal{Q}_{A h}\left[W_{A}\right]$ are the single-chain partition functions for a diblock copolymer and A homopolymer, respectively. These objects can be evaluated from a chain propagator $q(\mathbf{x}, s ;[W])$, which gives the statistical weight of observing a polymer of contour length $s$ at position $\mathbf{x}$. The propagator in turn can be computed by solving a modified diffusion equation as described below. $^{21,27}$

While the above field theory is formally exact, we have restricted our simulations to the mean-field limit of the theory - the so-called self-consistent field theory (SCFT). In SCFT, the partition function and average of an observable $G$ are approximated as:

$$
Z \approx \exp \left(-H\left[W_{+}^{*}, W_{-}^{*}\right]\right) \text { and } \quad<G\left[W_{+}, W_{-}\right]>\approx G\left[W_{+}^{*}, W_{-}^{*}\right] .
$$


where the dominant "mean-field" configurations $W_{ \pm}^{*}$ are obtained by solving the saddle point equations given by:

$$
\left.\frac{\delta H\left[W_{+}, W_{-}\right]}{\delta W_{ \pm}(\mathbf{r})}\right|_{W_{ \pm}^{*}}=0 .
$$

Since we work in the canonical ensemble, the Helmholtz free energy immediately follows from:

$$
\beta A=-\ln Z \approx H\left[W_{+}^{*}, W_{-}^{*}\right]
$$

\section{Numerical Methods}

The most expensive step in our SCFT simulations involves solving a modified diffusion equation for the copolymer chain propagator $q(\mathbf{x}, s ;[W])$ :

$$
\frac{\partial}{\partial s} q(\mathbf{x}, s ;[W])=\nabla^{2} q(\mathbf{x}, s ;[W])-W q(\mathbf{x}, s ;[W]), \quad 0 \leq s \leq 1
$$

subject to the initial condition $q(\mathbf{x}, 0 ;[W])=1$. A similar equation must be solved for the propagator of an A homopolymer. By discretizing $s$ with $N_{s}$ contour steps, the propagator at the $n+1$ contour step can be obtained by solving:

$$
q(\mathbf{x},(n+1) \Delta s ;[W])=e^{\Delta s \mathcal{L}} q(\mathbf{x}, n \Delta s ;[W])
$$

where $\mathcal{L}=\nabla^{2}-W$. As in the previous study by Bosse et al. ${ }^{21}$ this equation is solved on a discretized square domain with a spatial resolution of $\mathrm{dx}=\mathrm{dy}$ $=0.25$. Since the potential fields are diagonal on the collocation grid in real space and the Laplacian operator $\nabla^{2}$ is diagonal in Fourier space, we split the contour evolution operator as follows: ${ }^{40}$

$$
e^{\Delta s \mathcal{L}}=e^{-\Delta s W / 2} e^{\Delta s \nabla^{2}} e^{-\Delta s W / 2}+O\left(\Delta s^{3}\right)
$$

and perform the multiplication of the potential terms in real space, and the action of the Laplacian term in Fourier space. ${ }^{27,41}$ For the latter purpose, we used the FFTW fast Fourier transform (FFT) libraries. ${ }^{42}$ While the masking method with plane wave basis uses Discrete Fourier Transform (DFT), Discrete Since Transform of type I (DST-I) is used for the sine basis simulation. In the DST-I, collocation points are situated in the interior domain excluding the zero points at the boundaries. DST-I eliminates the need for complex data and utilize the symmetry of the data, hence speeds up the computation, 
while DST-I corresponds to the DFT of the odd-extended array of data. For example, a size- 4 DST-I of data $\{a, b, c, d\}$ corresponds to the size-10 DFT of the $\{-\mathrm{d},-\mathrm{c},-\mathrm{b},-\mathrm{a}, 0, \mathrm{a}, \mathrm{b}, \mathrm{c}, \mathrm{d}, 0\}{ }^{42}$

Solving the mean-field equations involves calculating the saddle point field configurations that satisfy the following stationary condition of the Hamiltonian:

$$
\begin{gathered}
\left.\frac{\delta H}{\delta i W_{+}}\right|_{W_{ \pm}^{*}}=\phi_{A c}(\mathbf{x})+\phi_{B}(\mathbf{x})+\phi_{A h}(\mathbf{x})-\phi(\mathbf{x})=0 \\
\left.\frac{\delta H}{\delta W_{-}}\right|_{W_{ \pm}^{*}}=\frac{2}{\chi N} W(\mathbf{x})_{-}^{*}-\frac{2 \chi_{w} N}{\chi N} \phi_{w}(\mathbf{x})-\phi_{A c}(\mathbf{x})+\phi_{B}(\mathbf{x})-\phi_{A h}(\mathbf{x})=0
\end{gathered}
$$

The saddle point configuration is obtained through a simple steepest descent search, adapted for a saddle point. Specifically, the fields are subjected to a relaxational dynamics along a fictitious 'time' variable $t$ with flow in the direction of the gradient of the Hamiltonian. The appropriate flow to locate a physical saddle point is in the direction of the gradient for the imaginary $W_{+}$field, and in the direction opposite to the gradient for the exchange $W_{-}$ field. Since we are interested only in the steady-state solution and not in the trajectory of the fictitious dynamics, stability is much more important than the accuracy of the time integration.

A simple algorithm, e.g. for the $W_{-}$field, is an explicit forward Euler update, given by:

$$
\frac{W^{(n+1)}-W^{(n)}}{\Delta t}=-\left.\frac{\delta H}{\delta W}\right|_{W^{(n)}}
$$

where the superscript $(n)$ denotes the $n^{t h}$ time step and $\Delta t$ is the discretized time step. However, due to its low stability threshold it does not allow us to use large time steps. Since our goal is to reach the steady-state as fast as possible, it is advantageous to consider a more-stable algorithm such as the semi-implicit-Seidel (SIS). ${ }^{30}$ The SIS scheme is given by:

$$
\frac{W^{(n+1)}-W^{(n)}}{\Delta t}=-\left[\left.\frac{\delta H}{\delta W}\right|_{W^{(n)}}-g * W^{(n)}\right]-g * W^{(n+1)}
$$

where $g * W$ is the linearized force, the kernel, $g$, is a Debye function, and $*$ denotes a convolution. The convolution is implemented in the $k$-space, thus 
$i W_{+}$update equation is given:

$$
i \hat{W}_{+}{ }^{(n+1)}=i \hat{W}_{+}{ }^{(n)}+\left.\left(\frac{\Delta t}{1+\Delta t \hat{g}(k)}\right) \frac{\widehat{\delta H}}{\delta i W_{+}}\right|_{i W_{+}^{(n)}}
$$

where $\hat{g}(k)$ is

$$
\begin{gathered}
\hat{g}(k)=\bar{\phi}\left\{\left(1-V_{A h}\right)\left(\hat{g}_{A A}+2 \hat{g}_{A B}+\hat{g}_{B B}\right)+\left(V_{A h} / \alpha\right) \hat{g}_{A h}\right\} \\
\hat{g}_{A A}(k)=\frac{2}{k^{4}}\left[f k^{2}+\exp \left(-k^{2} f\right)-1\right] \\
\hat{g}_{A B}(k)=\frac{1}{k^{4}}\left[1-\exp \left(-k^{2} f\right)\right]\left[1-\exp \left(-k^{2}(1-f)\right)\right] \\
\hat{g}_{B B}(k)=\frac{2}{k^{4}}\left[(1-f) k^{2}+\exp \left(-k^{2}(1-f)\right)-1\right] \\
\hat{g}_{A h}(k)=\frac{2}{k^{4}}\left[\alpha k^{2}+\exp \left(-k^{2} \alpha\right)-1\right]
\end{gathered}
$$




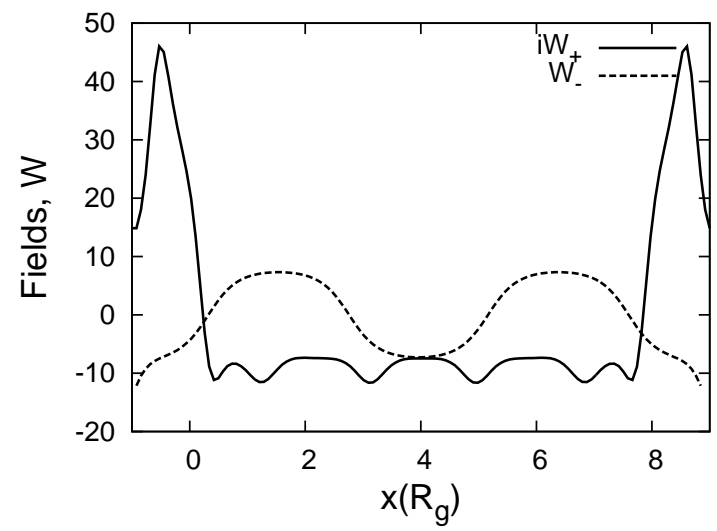

(a)

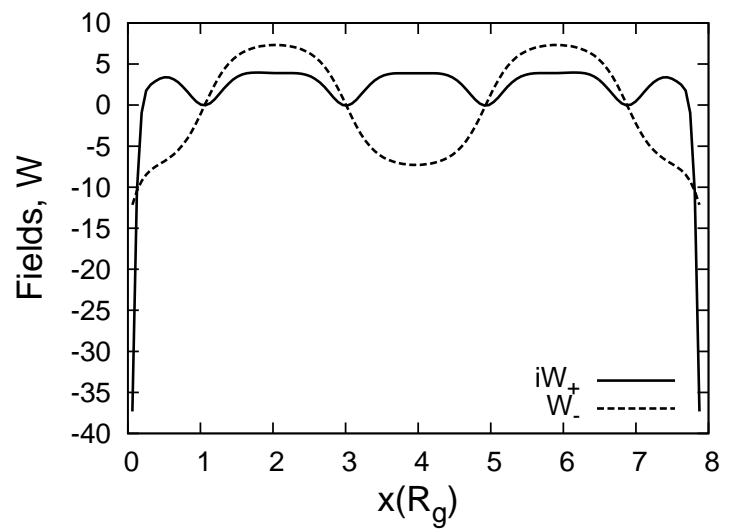

(b)

Figure 1: The converged $W_{ \pm}$field configurations over the one dimensional slab for (a) masking with a plane wave basis, and (b) sine basis simulations. 


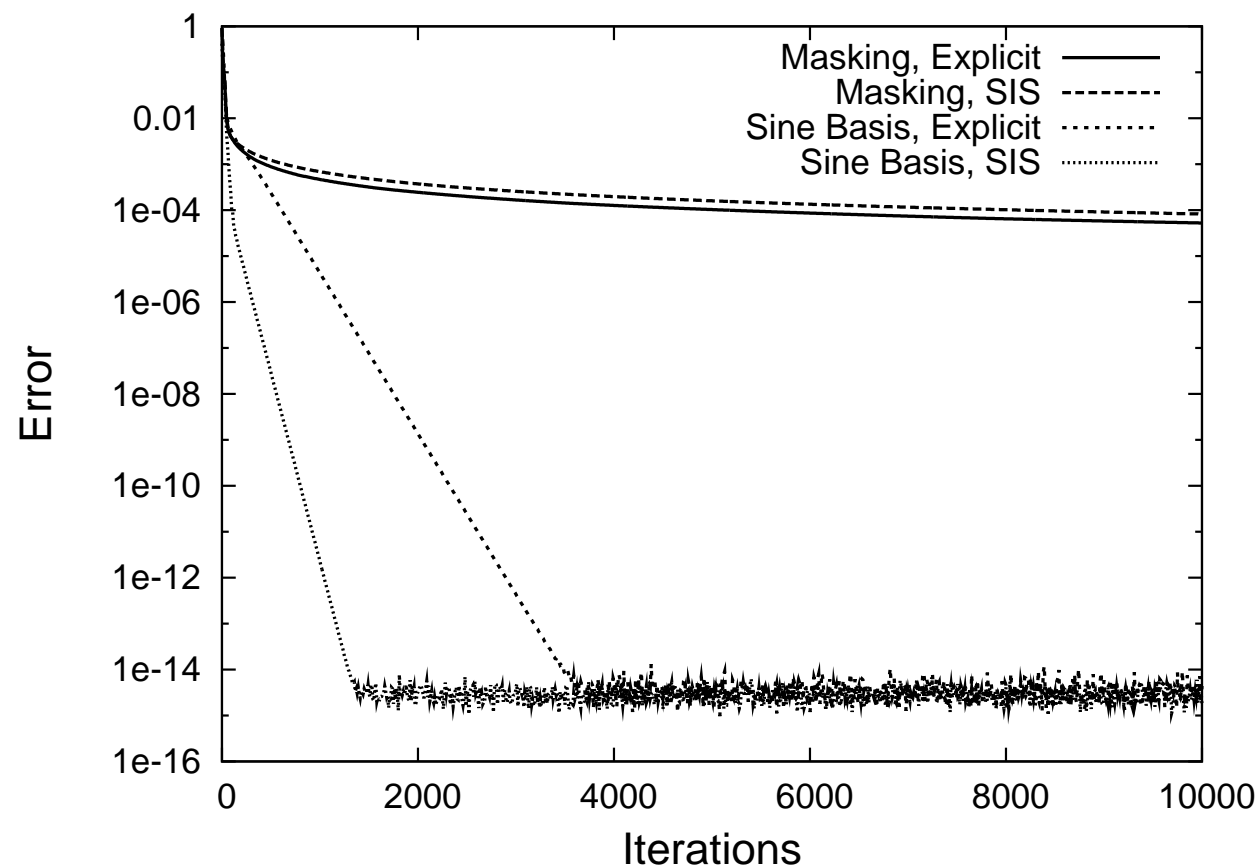

Figure 2: Plot of error, defined as the $l_{1}$ norm of the field-gradients of the Hamiltonian, as a function of field iteration steps for different numerical schemes. 

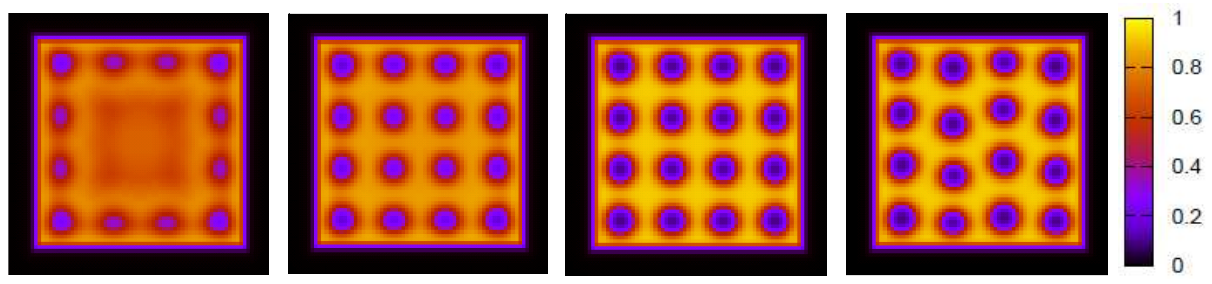

Figure 3: Representative density profiles (A segment volume fraction) of an $\mathrm{AB}$ diblock melt annealed within an A-attractive square well of side length $L=14 R_{g}$ after the $4000^{t h}(\chi N=13.75), 6000^{t h}(\chi N=15.75), 10000^{t h}$ $(\chi N=17)$ and $30000^{t h}(\chi N=17)$ field iteration. 

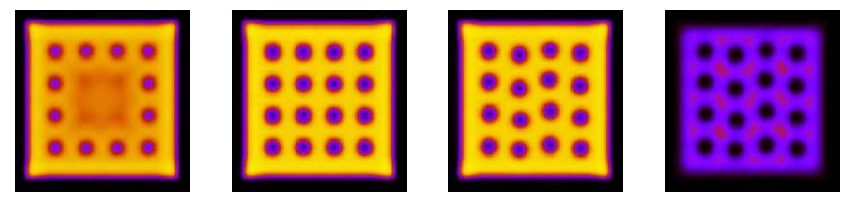

(a) Twisted hexagonal phase (HEX)
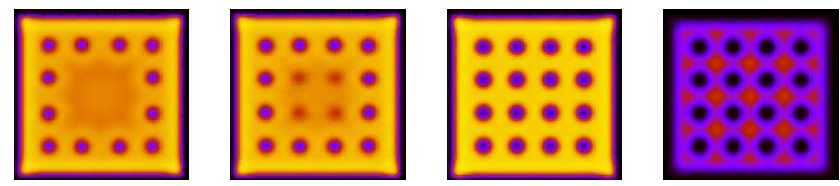

(b) Tetragonal phase (SQR)
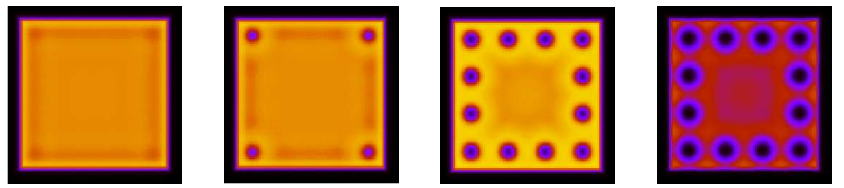

(c) Type I macroscopic phase separation (MAC I) for short A-homopolymer
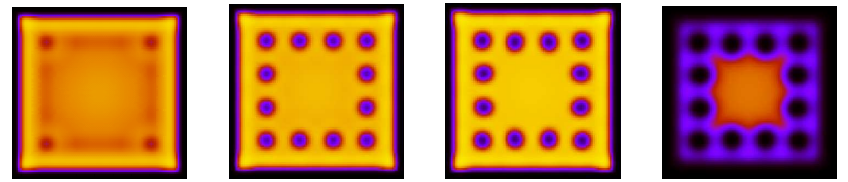

(d) Type I macroscopic phase separation (MAC I) for long A-homopolymer
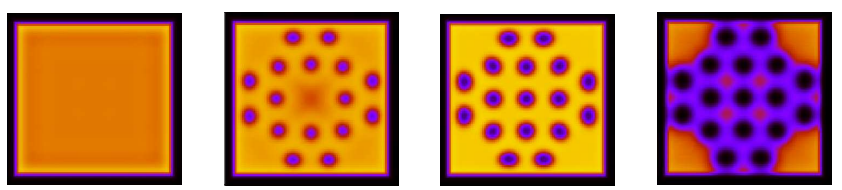

(e) Type II macroscopic phase separation (MAC II)
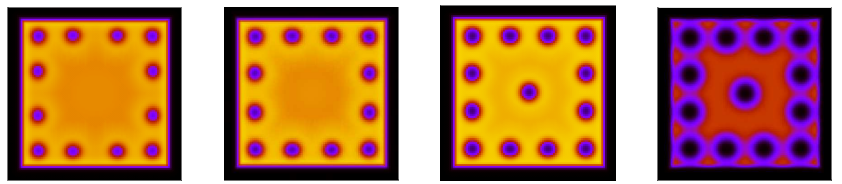

(f) Type III macroscopic phase separation (MAC III)

Figure 4: Evolution of ordering in SCFT simulations of AB block copolymer/A homopolymer blends with parameters (a) HEX: $\alpha / f=2.5, V_{A h}=$ $0.17, L=21 R_{g}$, B-wetting walls, (b) SQR: $\alpha / f=2.5, V_{A h}=0.23$, $L=23 R_{g}$, B-wetting walls, (c) MAC I: $\alpha / f=1.0, V_{A h}=0.4, L=17 R_{g}$, A-wetting walls, (d) MAC I: $\alpha / f=5.0, V_{A h}=0.2, L=22 R_{g}$, B-wetting walls, (e) MAC II: $\alpha / f=4.0, V_{A h}=0.33, L=21 R_{g}$, A-wetting walls, and (f) MAC III: $\alpha / f=2.8, V_{A h}=0.35, L=20 R_{g}$, A-wetting walls. The left three columns show the total A segmegt density at three subsequent stages of thermal annealing; the rightmost column shows the A homopolymer density in the resulting equilibrium structure. 


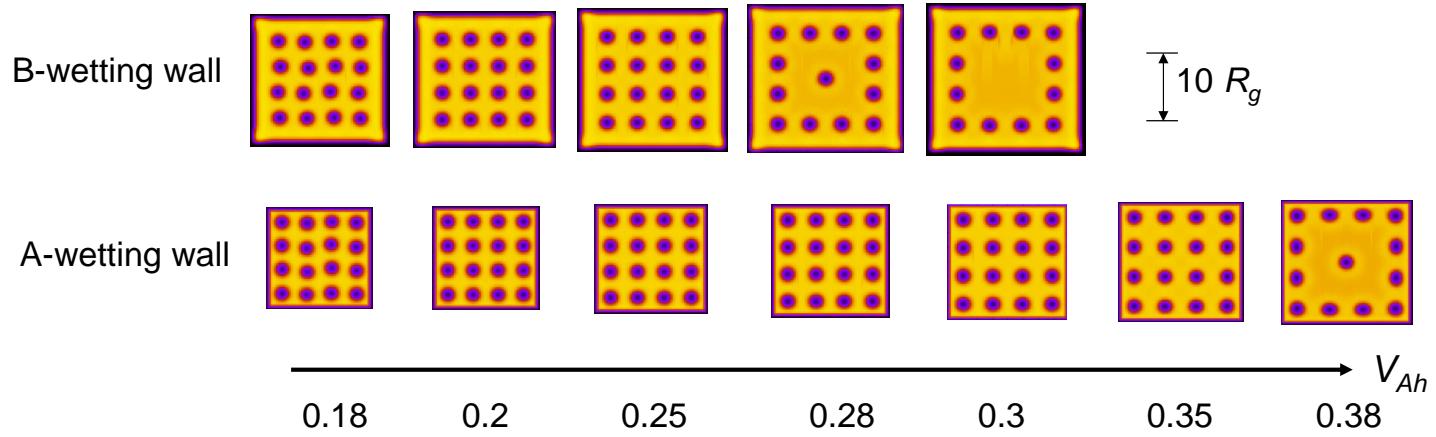

Figure 5: SCFT observed phases for a square-confined AB/A blend as a function of A homopolymer fraction $V_{A h}$ with both A- and B-wetting conditions and fixed ratio of homopolymer to diblock lengths, $\alpha / f=3.0$

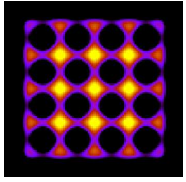

(a)

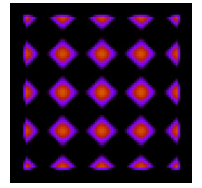

(b)

Figure 6: Representative A homopolymer volume fraction profiles, plotted in the range of $[0.3,0.6]$, at fixed $\alpha / f=3.0$ and (a) a B-wetting wall with $V_{A h}=0.25$ and $\mathrm{L}=24 R_{g}$, and (b) an A-wetting wall with $V_{A h}=0.25$ and $\mathrm{L}=17 R_{g}$. 


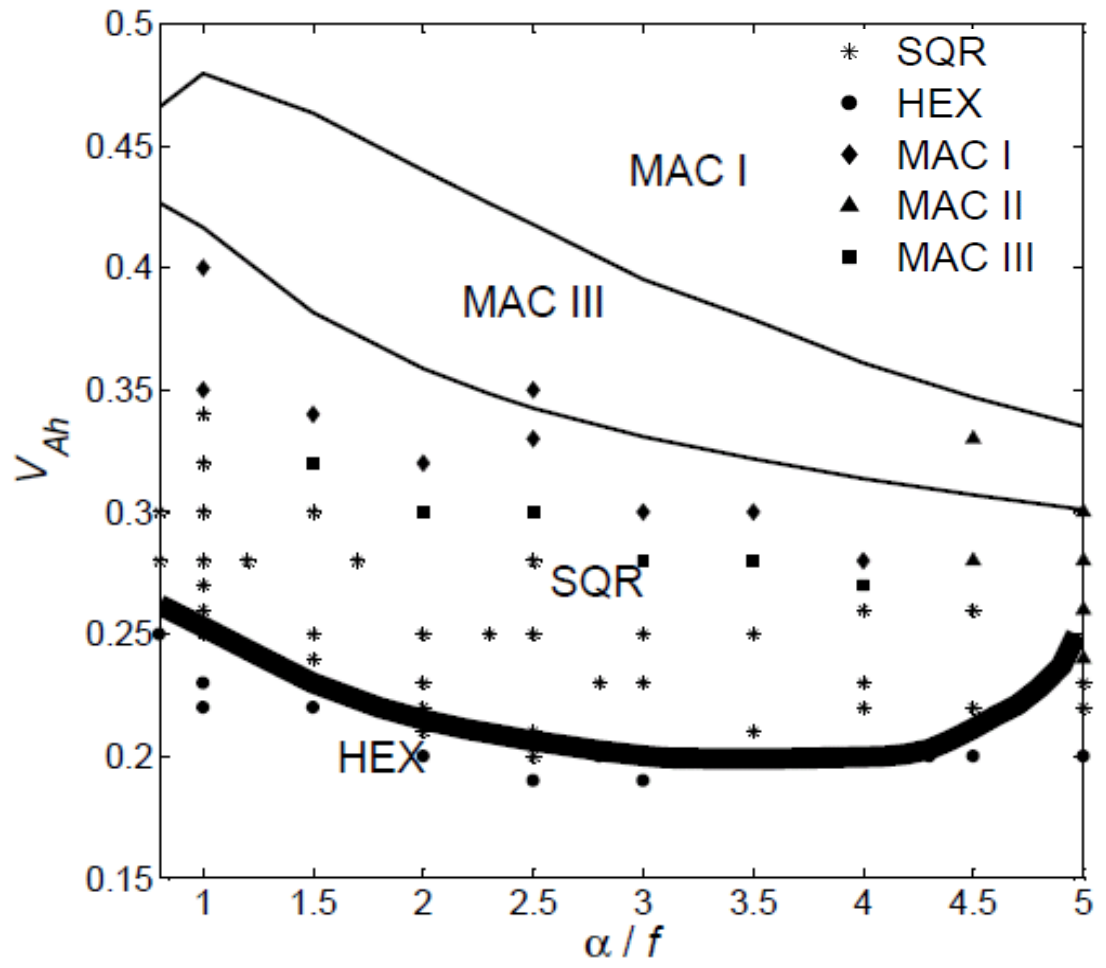

Figure 7: Phase diagram for an AB block copolymer/A homopolymer blend thin film subject to $17 R_{g} \times 17 R_{g}$ square confinement with A-wetting conditions. $\mathrm{x}$ - and $\mathrm{y}$-axis are ratio of homopolymer to diblock lengths $\alpha / f$ and $\mathrm{A}$ homopolymer fraction $V_{A h}$, respectively. 


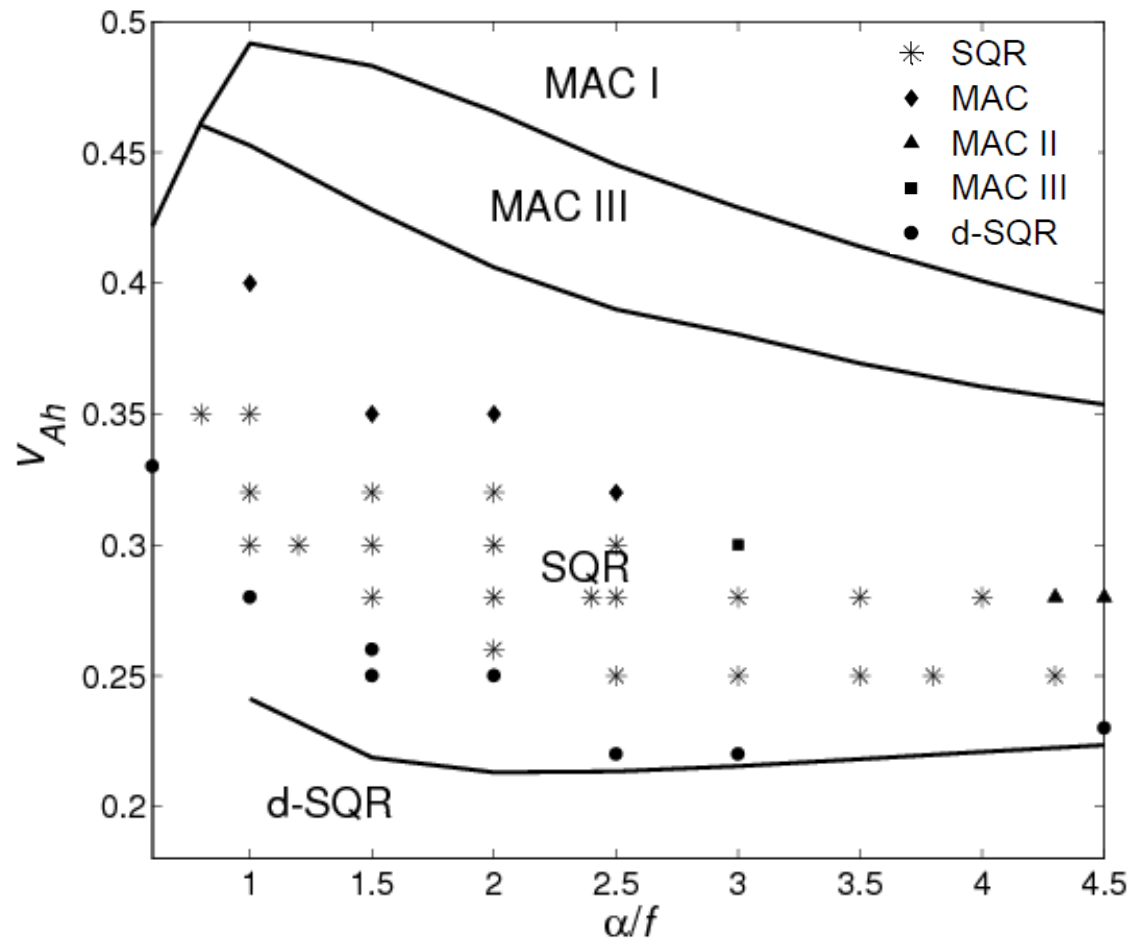

Figure 8: Phase diagram for an AB block copolymer/A homopolymer blend thin film subject to $18 R_{g} \times 18 R_{g}$ square confinement with A-wetting conditions. $\mathrm{x}$ - and $\mathrm{y}$-axis are ratio of homopolymer to diblock lengths $\alpha / f$ and $\mathrm{A}$ homopolymer fraction $V_{A h}$, respectively. 


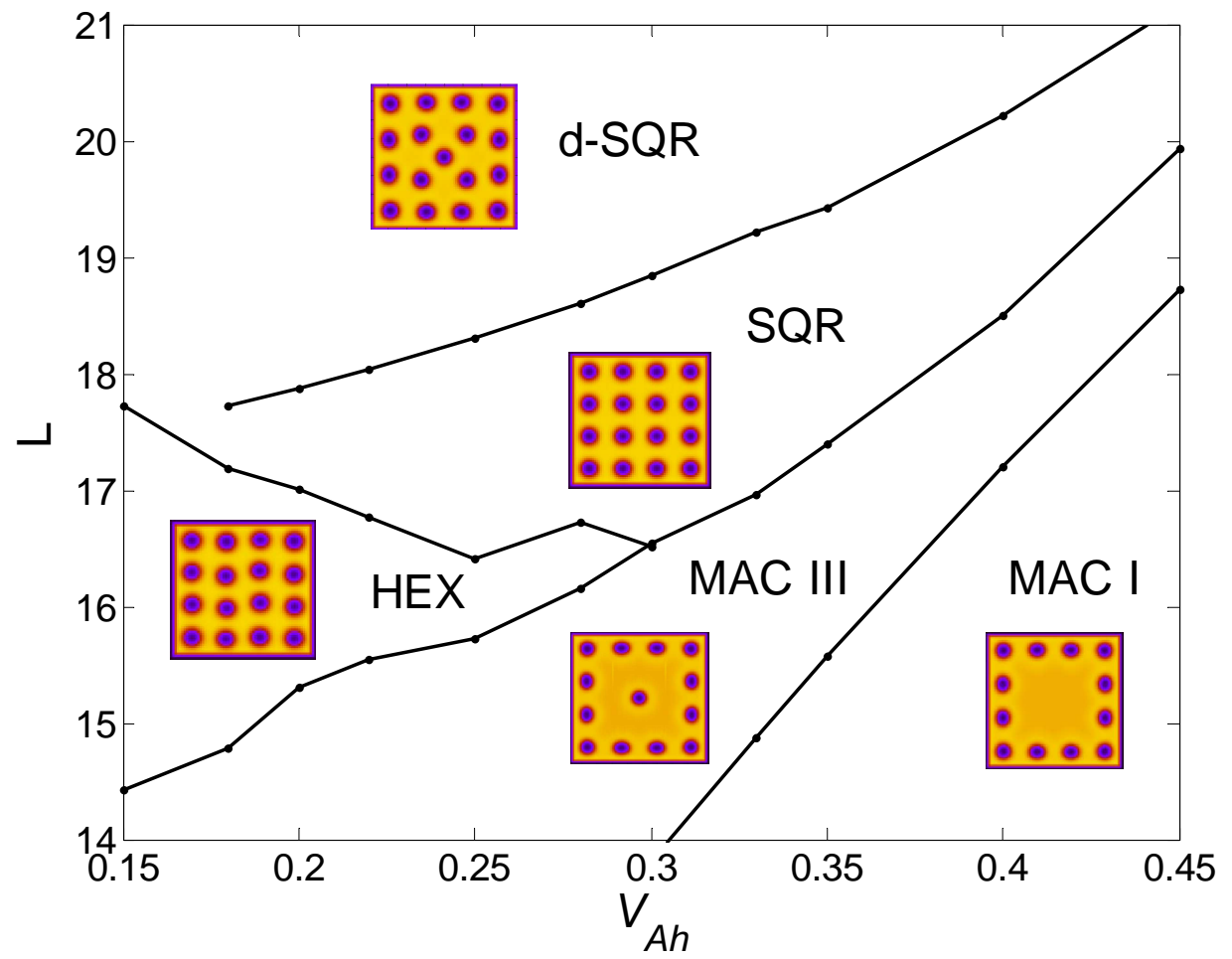

Figure 9: Phase diagram for an AB block copolymer/A homopolymer blend thin film subject to square confinement with A-wetting conditions and fixed ratio of homopolymer to diblock lengths, $\alpha / f=3.0$. $\mathrm{x}$ - and y-axis are $\mathrm{A}$ homopolymer fraction $V_{A h}$ and side length of square confinement $L$, respectively.

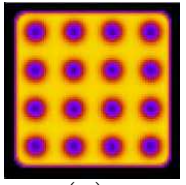

(a)

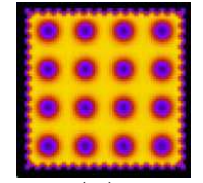

(b)

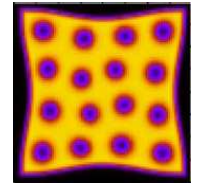

(c)

Figure 10: Representative volume fraction profiles of A segments under perimeter perturbations of square well confinement: (a)rounded corner, (b)high frequency perturbations, and (c)low frequency perturbations. 


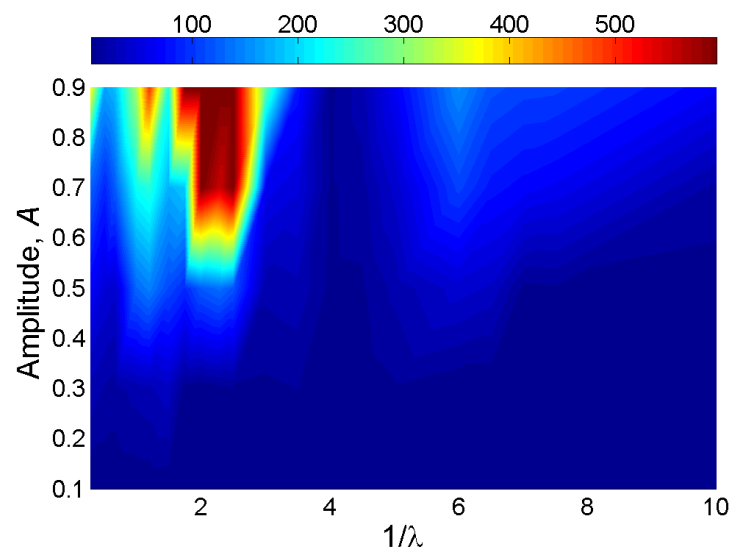

(a)

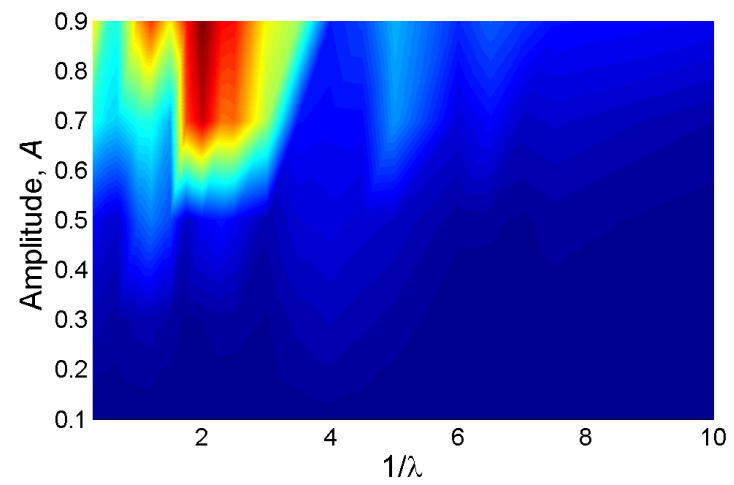

(b)

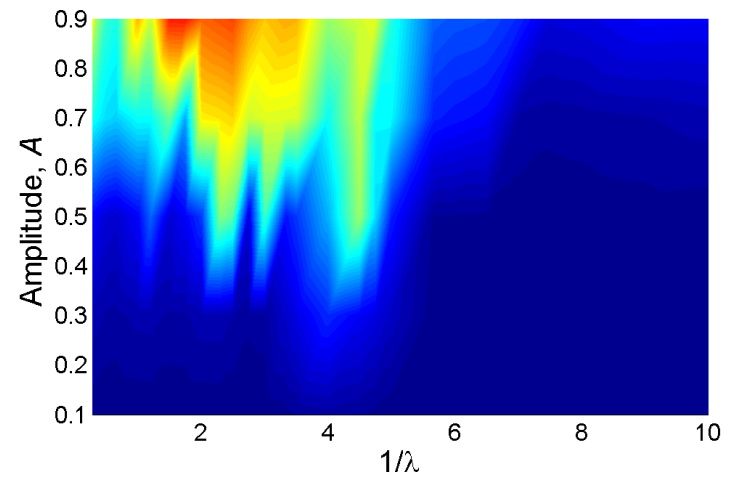

(c)

Figure 11: Intensity map of the order parameter, $\Psi$, of an AB block copolymer/A homopolymer blend film under harmonic perimeter perturbations of square well confinement in the spatiagl frequency $(1 / \lambda)$ and $\operatorname{amplitude}(A)$ with a phase shift $\Delta$ of (a) 0, (b) $0.5 R_{g}$ and (c) $1 R_{g}$. 


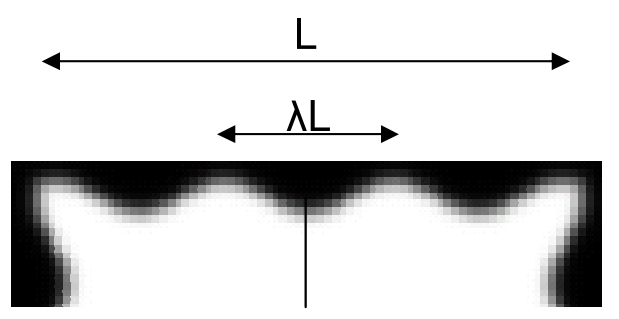

(a)

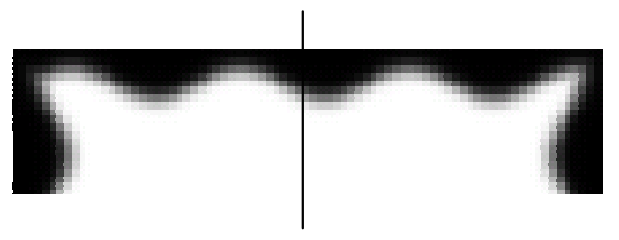

(b)

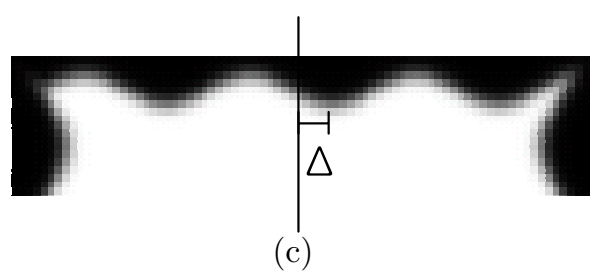

Figure 12: Representative density profile of square well confinement under harmonic perimeter perturbations $A \cdot \cos [2 \pi(x-\Delta) /(\lambda L)]$ of with $A=0.5 R_{g}$, $1 / \lambda=3$ and a phase shift $\Delta$ of (a) 0 , (b) $0.5 R_{g}$ and (c) $1 R_{g}$. 


\section{References}

1. Herr, D. J. C. Future Fab. Intl. Sec.5 2005, .

2. Mansky, P.; Chaikin, P.; Thomas, E. L. J. Mater. Sci. 1995, 30, 1987.

3. Park, M.; Harrison, C.; Chaikin, P. M.; Register, R. A.; Adamson, D. H. Science 1997, 276, 1401.

4. Harrison, C.; Park, M.; Chaikin, P. M. J. Vac. Sci. Technol. B. 1998, 16,544 .

5. Park, C.; Yoon, J.; Tomas, E. L. Polymer 2003, 44, 6725.

6. Segalman, R. A. Mater. Sci. Eng. R 2005, 48, 191-226.

7. Cheng, J. Y.; Ross, C. A.; Smith, H. I.; Tomas, E. L. Adv. Mater. 2006, 18, 2505.

8. Knoll, A.; Horvat, A.; Lyakhova, K.; Krausch, G.; Sevink, G. J. A.; Zvelindovsky, A. V.; Magerle, R. Phys. Rev. Lett. 2002, 89, 035501.

9. Stoykovich, M. P.; Nealey, P. F. Materials Today 2006, 9, 20.

10. Wilmes, G. M.; Durkee, D. A.; Balsara, N. P.; Liddle, J. A. Macromolecules 2006, 39, 2435.

11. Krishnamoorthy, S.; Hinderling, C.; Heinzelmann, H. Materials Today 2006, 9, 40.

12. Chen, H.; Chakrabarti, A. J. Chem. Phys. 1998, 108, 6897.

13. Wang, Q.; Nealey, P. F.; de Pablo, J. J. Macromolecules 2003, 36, 1731.

14. Segalman, R. A.; Hexemer, A.; Kramer, E. J. Phys. Rev. Lett. 2003, 91, 196101.

15. Sundrani, D.; Darling, S. B.; Sibener, S. J. Langmuir 2004, 20, 5091.

16. Cheng, J. Y.; Mayes, A. M.; Ross, C. Nature materials 2004, 3, 823.

17. Hammond, M. R.; Cochran, E.; Fredrickson, G. H.; Kramer, E. J. Macromolecules 2005, 38, 6575. 
18. Xiao, S.; Yang, X. M.; Edwards, E. W.; La, Y. H.; Nealey, P. F. Nanotechnology 2005, 16, S324.

19. Stein, G. E.; Kramer, E. J.; Li, X.; Wang, J. Phys. Rev. Lett. 2007, 98, 086101.

20. Bita, I.; Yang, J. K. W.; Jung, Y. S.; Ross, C. A.; Thomas, E. L.; Berggren, K. K. Science 2008, 321, 939.

21. Bosse, A. W.; García-Cervera, C. J.; Fredrickson, G. H. Macromolecules 2007, 40, 9570 .

22. Park, S.; Craig, G. S. W.; La, Y.; Solak, H. H.; Nealey, P. F. Macromolecules 2007, 40, 5084 .

23. Matsen, M. W. J. Chem. Phys. 1998, 108, 785.

24. Tang, C.; Lennon, E. M.; Fredrickson, G. H.; Kramer, E. J.; Hawker, C. J. Science 2008, 322, 429.

25. Stoykovich, M. P.; Müller, M.; Kim, S. O.; Solak, H. H.; Edwards, E. W.; de Pablo, J. J.; Nealey, P. F. Science 2005, 308, 1442.

26. Fredrickson, G. H.; Ganesan, V.; Drolet, F. Macromolecules 2002, 35, 16.

27. Fredrickson, G. H. The Equilibrium Theory of Inhomogeneous Polymers; Clarendon Press: Oxford, 2006.

28. Park, S.; Stoykovich, M. P.; Ruiz, R.; Zhang, Y.; Black, C. T.; Nealey, P. F. Adv. Mater. 2007, 19, 607.

29. Matsen, M. W. J. Chem. Phys. 1997, 106, 7781.

30. Ceniceros, H. D.; Fredrickson, G. H. Multiscale Model. Simul. 2004, 2, 452.

31. Matsen, M. W. Phys. Rev. Lett. 1995, 74, 4225-4228.

32. Fredrickson, G. H. Macromolecules 1987, 20, 2535.

33. Brown, G.; Chakrabarti, A. J. Chem. Phys. 1994, 101, 3310. 
34. Harrison, C.; Chaikin, P. M.; Huse, D. A.; Register, R. A.; Adamson, D. H.; Daniel, A.; Huang, E.; Mansky, P.; Russell, T. P.; Hawker, C. J.; Egolf, D. A.; Melnikov, I. V.; Bodenschatz, E. Macromolecules 2000, 33, 857.

35. Cheng, J. Y.; Zhang, F.; Smith, H. I.; Ross, C. A. Adv. Mater. 2006, $18,597$.

36. Brueck, S. R. J.; Chen, X. J. Vac. Sci. Technol. B 1999, 17, 908.

37. Park, S.; Park, O.; Cheng, J. Y.; Rettner, C. T.; Kim, H. Nanotechnology 2008, 19, 455304.

38. Leibler, L. Macromolecules 1980, 13, 1602.

39. Alexander-Katz, A.; Moreira, A. G.; Fredrickson, G. H. J. Chem. Phys. 2003, 118, 9030 .

40. Tzeremes, G.; Rasmussen, K. K.; Lookman, T.; Saxena, A. Phys. Rev. E 2002, 65, 041806.

41. Boyd, J. P. Chebyshev and Fourier Spectral Methods; Dover: Mineola, 2000.

42. Frigo, M.; Johnson, S. G. 2006 FFTW manual for version 3.1 .2 found at http://www.fftw.org. 
For Table of Contents Use Only

Title : SCFT Simulations of Thin Film Blends of Block Copolymer and Homopolymer Laterally Confined in a Square Well

Authors : Su-Mi Hur, Carlos J. García-Cervera, Edward J. Kramer and Glenn H. Fredrickson
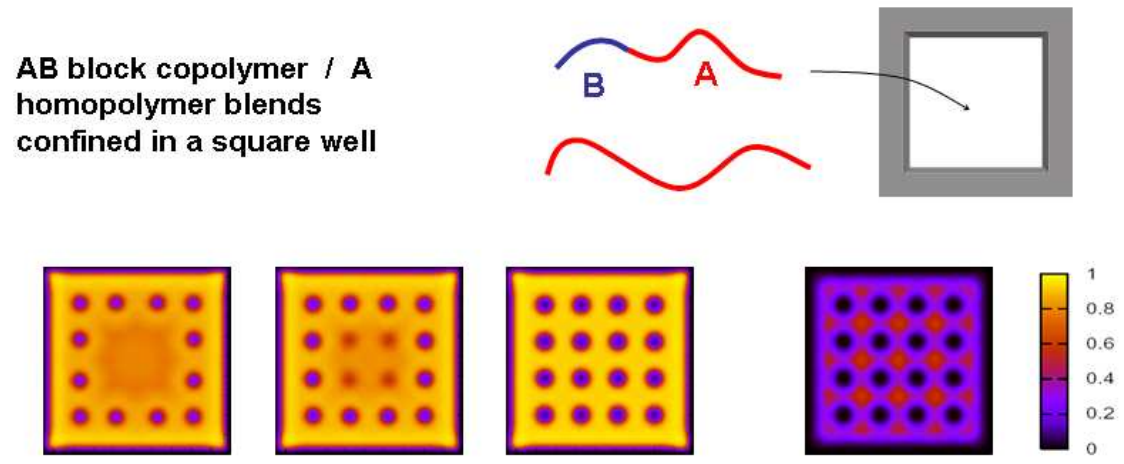

Evolution of total A-segment density

A homopolymer localization stabilizes tetragonal order 